\title{
The Secret of Human Existence Homeostasis: Spiritual Intelligence is the Hope of All Humanity
}

\author{
Boshra A Arnout ${ }^{1}$ and Ahed J Alkhatib ${ }^{2,3 *}$ \\ ${ }^{1}$ Department of psychology, Jordan \\ ${ }^{2}$ Department of Legal Medicine, Toxicology of Forensic Science and Toxicology, School of Medicine, Jordan University of Science and Technology, Jordan \\ ${ }^{3}$ International Mariinskaya Academy, department of medicine and critical care, department of philosophy, Academician secretary of department of Sociology, \\ Jordan
}

*Corresponding author: Ahed J Alkhatib, Department of Legal Medicine, Toxicology of Forensic Science and Toxicology, School of Medicine, Jordan University of Science and Technology, Jordan.

Received Date: July 16, 2019

Published Date: July 29, 2019

\begin{abstract}
This study continues our descriptive and experimental studies addressing spiritual intelligence. As Zohar \& Marshall pointed out that spiritual intelligence helps us to live life at a deeper level of meaning. We can also use the skills of spiritual intelligence (SQ) to deal with the problems of good and evil, life and death problems, and to help us understanding more deeply the causes of human suffering. Human beings are the only biological beings with spiritual intelligence. It has a capacity for harmony with oneself and others. Scientists believe that neither IQ nor social intelligence can adequately explain the complexity of the broad intelligence of the human mind. From the previous literature and studies about spiritual intelligence and neural basis, it is plausible to argue that through training programs to develop spiritual intelligence skills, we can modify the activity of the autonomic nervous system and increased control over its activities. The central component of any program to relief mental disorders and physical health problems is the development of the spiritual intelligence skills, which may be the hope of alleviating the suffering of patients in hospitals. We recommend the necessity and importance of applying the Biopsychosocial-Spiritual Model in all institutions that provide health care to patients with physical and psychological illnesses.
\end{abstract}

Keywords: Homeostasis; Spiritual intelligence; Biological basis; Mortality; Pain symptom Manage; Selye stress theory

\section{Introduction}

Positive psychology is based on a theory that stems from how the individual learns and how achieves a healthy, convincing, good and enjoyable life. The positive, and its commitment to care and development, leads to the understanding of the human being, the world and others around him, and even changes his negative thinking towards life as a whole. Positive psychology has defined as the scientific study of the strengths and virtues that empower individuals, institutions and societies to flourish. This has been based on the belief that human beings wish to lead a human life full of value and meaning in which they realize their ambitions and employ In which their human abilities to reach true satisfaction and happiness, and improve their general humanitarian experience in all areas of human value such as social relations, work, and even play [1].
Perhaps one of the most important psychological strengths in humans is intelligence, which has received a great deal of attention from psychologists over time, and even considered the IQ is the first factor of success and excellence, and that the highest people in intelligence reach the highest levels of success in education, work and various areas But scientists have recently discovered that other types of intelligence play an important role in success, especially in reality. Many intelligent people stumble and spend their lives in fear and tension, and others less intelligent take important and successful positions in life [2].

This means that intelligence is not only attributed to words, numbers, and logic, but also numerous types of intelligence have been described. There are creative, personal, sensory, physical, sexual, spiritual and spiritual intelligence, as well as numerical and 
verbal intelligence. Spiritual intelligence, as Amram \& Dryer [3], is the ability to apply and use spiritual abilities and qualities that increase our effectiveness in life. Arnout [4] argued that if emotional intelligence is correlated between mind and emotion, Spiritual Intelligence is the most problematic of psychology schools, from psychoanalysis to knowledge, and equates man as being body, mind and spirit together.

Buzan [5] mentioned that Spiritual intelligence excels and competes with multiple intelligence and emotional intelligence to form a trilogy of excellence, success, and excellence. Many people consider spiritual intelligence the most important intelligence, with its power capable of changing their lives, civilizations, the course of history, and the entire planet.

Arnout [6] pointed out that with these rapid changes, the human being of the twenty-first century is exposed to many disasters and crises of physical, economic, environmental, natural, social and psychological, which makes a lot of stock of strength, will and hardness may be dispersed in the midst of these stressors, which is impossible to stop, but only to cope them effectively and positively.

\section{The Biological Basis of Spiritual Intelligence}

Thorndike explains biological intelligence: that intelligence and mental processes as a result of the work of a complex neurological system perform a function in a completely different way. This means that intelligence - as Thorndike goes - is determined by latent possibilities in the physical configuration of the organism inherited and not acquired, and the more complex the nervous system of the organism the more its intelligence. Gardner emphasized that there is a physiological and biological basis for the types of intelligence, and that the human brain is divided into two differentiated or asymmetrically differentiated anatomical. This anatomical variation is analogous to functional differences that reflect the difference of functions between the two parts.

Both Zohar \& Marshall [7] noted that 40 Hertz were observed from the waves across the brain, and that these vibrations were linked to the ability of consciousness, and that they communicated cognitive events and cognitive comprehension During a more extensive gloss, and went on to say that these waves represent the neural base of spiritual intelligence. This is confirmed by Emmons [8] the data of spiritual intelligence collected neurological, evolutionary, and psychological evidence was consistent with Gardner's criteria.

Since spiritual intelligence is the comprehensive intelligence of all intelligences, it was found that its components and dimensions have biological bases and in multiple locations of the brain, and will be presented as follows:

- After the overall picture and intuition, there was a correlation between mental processes such as language and logical processes with the left hemisphere of the brain, whereas the perception of the overall picture and intuition is linked to the right half [9]
- $\quad$ After sympathy and cooperation, Gallese [10] found that discoveries implying that biological research confirmed that the neuron system is a physiological basis for empathy and cooperation.

- Sublimation: Hamer [11] found that genes also play an important role in determining the ability to transcend the spiritual intelligence, which indicates that special processes occur in certain regions of the brain is responsible for this form of intelligence.

- Symbolic imagination and its role in intellectual evolution suggested that the brain has the ability to build symbolic figurative processes which led to profound reflective thinking about the meaning of life, experience, and human existence.

- $\quad$ Search for the meaning of life: At the end of 1990, it has been announced the neuroscientists have discovered the socalled God Spot God in the brain, a mass of nerve tissue in the temporal lobe of the brain, and this block to make the person raises the fundamental questions of meaning and existence, and make it Looking for substantive answers, optimal solutions to problems, making him aspire to the highest things to a dream of better growth, and being active when he has spiritual experiences "a deep sense of love". The monitoring of nerve oscillations has led to a third type of thinking that results in cognitive processes that answer the questions of significance.

- Meditation: Studies found that those who were trained in meditation showed significantly greater activity in the cerebral cortex of the frontal and clear effects on the brain and immune functions. Lazar and his colleagues [12] found that the practice of meditation is associated with increased thickening of the cerebral cortex. It was found that meditators and dummies are the highest and thickest of these areas of the brain, while empathy and meditation are found to change electrical patterns in the brain, suggesting that the development of the qualities of spiritual intelligence, especially (sympathy and love) involves complementary mechanisms that may lead to short and longterm neurological changes in the brain.

- The skills of humor and spontaneity: Some researchers found that the skills of language humor, represented in the ability to recognize the pleasant and sarcastic hints (one of the components of spiritual intelligence), and ability to understand the interpretations of the metaphorical language - part of the function of the right hemisphere - which can be important in understanding the methods of ridicule and metaphor, so patients with right hemisphere tend to understand language in a literal manner.

The individual who has suffered damage to the Broca region may have substantial damage to linguistic intelligence and difficulty in speaking, reading, and writing, but he will still be able to sing, solve mathematical problems, meditate on feelings, and relate to others. When we use our minds for thought, this process involves not only the brain and the intelligent outcome, but also emotional 
or emotional intelligence, as well as spirituality, values, hopes and feasibility. The neurotransmitters in the brain are dedicated tolinking mental, emotional, and spiritual experiences. Thus, we believe that spiritual intelligence works to activate the two hemispheres of the brain together. It performs functions from the right hemisphere as well as the left. This supports the "representative of intelligence" and refers to the integration of all other intelligences in spiritual intelligence and has a clear biological basis.

Singer \& Gray [13] found that neurotransmitters in the brain are dedicated to connecting mental, emotional and spiritual experiences. Where the thinking in the scientific community that the brain is only able to produce two types of processes are: neurological processes that allow the brain to do rational and logical thinking, and the second type of nervous processes connecting thousands of neurons and the resulting emotional ideas. Neural oscilloscopes have led to a third type of thinking that results in cognitive processes that answer the questions of significance.

Deacon [14] examined the evolution of symbolic imagination and its role in mental development. He found that the brain has the ability to build symbolic processes of imagination that result in profound contemplative thinking about the meaning of life, life experiences, and human existence.

Zohar \& Marshall [8] pointed out that the spiritual intelligence operates from the center of the brain (or the functions of the unified neuroscience). It integrates and harmonizes all kinds of intelligence. Spiritual intelligence makes us rational, emotional and spiritual beings integrated, which God has created in reality. All three types of intelligence (mental - emotional - spiritual) work together complementing each other, and our brains designed by the Creator so that they can achieve this integration. Every intelligence of these intelligences has its own area of strength in the brain, and it can function independently of other types of intelligences. That is, we do not have high or low abilities for the three types of intelligence at the same time. The individual does not need to have a great mental or spiritual intelligence to have high emotional intelligence, and the individual may possess a high intellectual intelligence but less emotional intelligence and spiritual both, and so on.

Since 1999, Gardner has emphasized the existence of a physiological and biological basis for the types of intelligence, and classical cognitive theories in the interpretation of intelligence have confirmed the association of mental processes such as language and logical processes to the left half of the brain. Perception of the overall picture and intuition (which are the two main components of intelligence) Spiritual) are associated with the right hemisphere.

Davison \& his colleagues [15] found that those who received relaxation exercises to increase vigilance and meditation showed significant extra activity in the front of the cortex during training compared to other times. Lutz, et al. [16] found that the development of spiritual intelligence skills is associated with long-term and short-term changes to specific areas of the brain. Gallese [10] found that discoveries and biological research confirm that the Neuron System is a physiological basis for empathy and cooperation, which are spiritual intelligence skills. Also, Homer [11] found that genes also play an important role in determining the ability to excel.

We can say that the results of biological and neurological studies indicate that there is a relationship between the abilities and skills of spiritual intelligence, such as self-sufficiency, total thinking, perception of the whole picture, intuition, empathy, honesty, love and vigilance, and that these spiritual abilities have a biological basis associated with the occurrence of special processes In specific regions of the brain, as well as the possibility of genetic factors.

Sulmasy [17] noted that in "the Biopsychosocial-Spiritual Model of Care, everyone, according to this model, has a spiritual history. For many persons, this spiritual history unfolds within the context of an explicit religious tradition. But, regardless of how it has unfolded, this spiritual history helps shape who each patient is as a whole person, and when life-threatening illness strikes, it strikes each person in his or her totality. This totality includes not simply the biological, psychological, and social aspects of the person, but also the spiritual aspects of the whole person as well. This biopsychosocial-spiritual model is not a "dualism" in which a "soul" accidentally inhabits a body. Rather, in this model, the biological, the psychological, the social, and the spiritual are only distinct dimensions of the person, and no one aspect can be disaggregated from the whole. Each aspect can be affected differently by a person's history and illness, and each aspect can interact and affect other aspects of the person.

If schizophrenia caused by increased activity of a neurodegenerative neurotransmitter in the brain, dopamine, and mania from increased norepinephrine or dopamine secretion, while depression from reduced norepinephrine activity, we argued that interventions based on spiritual intelligence and education of individuals suffering from these mental disorders of spiritual intelligence skills, to control the levels of chemical neurotransmitters in the brain?. Is it possible for spiritual intelligence interventions to balance the neural conductors and increase the effectiveness of drugs, especially as mental patients are still as they are in hospitals and the disorder state has not improved as needed? Can spiritual intelligence interventions mitigate the violent behavior of prisoners and criminals by affecting brain cells and parts responsible for emotions and behavior? Can spiritual intelligence interventions mitigate the violent behavior of prisoners and criminals by affecting brain cells and brain parts responsible for emotion and behavior? It can even reduce the frequency of obsessive-compulsive disorder patients in frequent self-destructive behaviors?.

And is the spiritual intelligence interventions benefit for addicted people to reduce their psychological dependence on drugs. Do spiritual intelligence interventions benefit to increased hope, optimism, forgiveness, positive attitude, gratitude, love of life, psychological well-being and the quality of life of chronically patients with cancer, kidney failure, HIV / AIDS and others? Do spiritual intelligence interventions help to increase the age of the elderly, their sense of happiness and achievement, and reduce their anxiety and death? Do spiritual intelligence interventions improve 
the mental, physical and psychological functioning of children with special needs? and is also help in achieving the normal path of physical, physiological, mental and social growth of ordinary children? These hypotheses need many experimental studies and will have many applications. It is considering a huge breakthrough in medical and psychological therapy if true.

\section{Spiritual Intelligence and Human Existence Homeostasis}

As mentioned by Jain and Purohit [18], spiritual intelligence is more than individual mental skill. In addition to self-awareness, it implies awareness of our relationship, it concerned with the internal life of mind and spirit and its association with being in the world. It implies a capability for a deep understanding of existential questions and insight into multiple levels of consciousness.

The concept of spiritual intelligence is related to human nature or what we call instinct. The nature of man is spirituality, religiosity and goodness, and therefore this nature must be preserved to organize human life. And commitment to worship as a way of life, and adherence to ethics that will sharpen the personality of man and strengthen the relationship between the servant and his Lord, and also between him and others, and increase the awareness of the person and the world and the whole universe, which leads to a balance in the life of the individual in the light of the standards and provisions.

Spirituality, as Wigglesworth [19] sees is "the innate human need to connect with something greater than ourselves, spirituality has always been seen by humanity as one of the fundamental requirements as it embodies the highest levels of cognitive, moral and emotional development and attempts to answer life questions [7] also argues with Emmons that spirituality represents a distinctive way of adaptation and represents a different understanding of science of values and goals in life. Thus Natti (2008) pointed out that spiritual intelligence is an essential capacity that shapes and guides all other abilities.

In his study of cognitive growth, Piaget pointed out that intelligence is an aspect of an individual's adjustment to his environment and involves the interaction between two mental processes of assimilation and harmony. Baldwin [20] refereed that Piaget's theory of cognitive development is a comprehensive theory of the nature and evolution of human intelligence. Piaget's idea is originally conceived as the theory of the developmental stage. One of the basic concepts in this theory is the idea of assimilation and residence. Assimilation means how people perceive and adapt to new information and adapt new information to old cognitive schemes. In this process, new experiments are adapted to or similar to old ideas. Piaget emphasized that the human mind is programmed to harmony, which is believed to be what ultimately affects the structures through internal and external processes during the process of assimilation and representation.

From this point of view, Human homeostasis occurs as a result of the individual's encounter with problems and difficulties and cannot solve them through his limited past experiences. In order to regain its equilibrium, it must undertake a structured and knowledgeable activity to seek new knowledge that will enable him to solve these problems and develop his / her cognitive structure and remove the tension and anxiety it has suffered.

According to the WHO [22], health is defined as a positive concept emphasizing social and personal resources, as well as physical capabilities. This stressed the important of identifying the health risks associated with different behavioral and social conditions and not just the biological illness itself or the medical factors only.

Because, as Foglio and Brody [19] mentioned "patients while struggle with the physical aspects of their disease, they have other pain as well: pain related to mental and spiritual suffering, to an inability to engage the deepest questions of life. Patients may be asking questions such as the following: Why is this happening to me now? What will happen to me after I die? Will my family survive my loss? Will I be missed? Will I be remembered? Is there a God? If so, will he be there for me? Will I have time to finish my life's work? One physician who worked in the pediatric intensive care unit told me about his panic when his patients' parents posed such questions. It is difficult to know what to say; there are no real answers. Nevertheless, people long for their physicians as well as their families and friends to sit with them and support them in their struggle. True healing requires answers to these questions.

From the previous view, biopsychosocial illness is possibly caused by excess stress. Lazarus and Folkman [23] pointed out that stressors are the situations in which individuals are faced with environmental or other demands which exceed their immediate ability to cope. Thus, we argued a Bio-Psychosocial-Spiritual Model which help healthcare providers to understanding and illness or problems of the person and formation of treatment plan. Shapiro [24] said that "change is constant. That's the truth of being alive. Our bodies are constantly shifting, growing, shrinking, developing new set points for homeostasis, accommodating new information. Whether we look or not, this process is happening in every moment. When I lost my illusion of permanence and began actually to look at what life was offering to me, I discovered a wealth of possibilities I had never stopped to notice. I was stunned at the abundance. That abundance had been there all along. I had simply never looked".

Thus, we illustrate the importance of spiritual intelligence in human health and homeostasis, from the results of studies by physicians and psychologists which research about the relations between spiritual intelligence and each of mortality and pain symptom manage.

\section{Spiritual intelligence and mortality}

The study of Strawbridge, Cohen, Shema and Kaplan [14] aimed to analyze the relationship between religious attendance and mortality. Results found that Lower mortality rates for frequent religious attenders are partly explained by improved health practices, increased social contacts, and more stable marriages occurring in conjunction with attendance. At the same time, the study conducted by Oman and Reed [24] that aimed to test the 
prospective relationship between attending religious services and all-cause mortality to determine, the results of analysis shown that lower mortality rates for those who attend religious services are only partly explained by the six possible confounders: demographics, health status, physical functioning, health habits, social functioning and support, and psychological state.

Li, Stamofer, Williams and VanderWeele examined the association between attendance at religious services and mortality, results illustrated that frequent attendance at religious services was associated with significantly lower risk of all-cause, cardiovascular, and cancer mortality among women. Religion and spirituality may be an underappreciated resource that physicians could explore with their patients, as appropriate. As well Williams and Sternthal [25] emphasized that health practices and social ties are important pathways by which religion can affect health. Other potential pathways include the provision of systems of meaning and feelings of strength to cope with stress and adversity. Thus D'Souza [26] argued that doctors and clinicians should not "prescribe" religious beliefs or activities or impose their religious or spiritual beliefs on patients. The task of in-depth religious counselling of patients is best done by trained clergy. In considering the spiritual dimension of the patient, the clinician is sending an important message that he or she is concerned with the whole person. This enhances the patientphysician relationship and is likely to increase the therapeutic impact of interventions. Doctors, health care professionals and mental health clinicians should be required to learn about the ways in which religion and culture can influence patients' needs and recovery.

Wilding [27] qualitative study found that in the past 1020 years there has been increasing interest in the relationship between spirituality and health. he interviewed six patients from community mental health centers, using a phenomenological approach to explore how concepts of spirituality, occupation and mental illness/mental health are related. Four main themes were identified: Spirituality is a phenomenon that provides meaning to life. Spirituality can help a person cope with mental illness. Spiritual beliefs can make everyday occupations more meaningful and health-enhancing. Some people find it valuable to engage in shared occupations that focus on spirituality. Spirituality is an important and relevant issue to be discussed between patients and health practitioners, provided that practitioners can exercise sensitivity, caution, tolerance and acceptance of values that may differ from their own".

Therefore, Winslow and Wehtje-Winslow [28] mentioned that "in an age that features technologically sophisticated medical interventions, patients still desire spiritually nurturing health care. Attention to patients' spiritual needs and resources in the clinical setting may raise a number of ethical questions. Five ethical guidelines are offered as illustrations of norms that respect patients' preferences and preserve health care professionals' integrity".

The study conducted by Gioiella, Berkman and Robinson [29] support the inclusion of spirituality as part of routine patient assessment and intervention. Clinical intervention that would increase a patient's level of spiritual awareness and his or her level of comfort associated with a personal perspective on death could help decrease the patient's level of psychosocial distress. Despite the medical establishment's bias to the contrary, religion and spirituality are positively associated with both physical and mental health and may be particularly significant to terminally ill patients. The curricula of medical, nursing, and other health schools should be redesigned appropriately.

Koslander, da Silva, et al. [30] illuminated how existential needs and spiritual needs are related to health care ethics and individuals' mental health and well-being. The term existential needs is defined as the necessity of experiencing life as meaningful, whereas the term spiritual needs is defined as the need of deliverance from despair, guilt and/or sin, and of pastoral care. It discusses whether or not patients' needs are holistically addressed in Western health care systems that neglect patients' existential and spiritual needs, because of their biomedical view of Man which recognizes only patients' physical needs. It excludes a holistic health care which considers all needs, expressed by patients in treatment of mental illness. Addressing all needs is important for patients' improvement and recovery. For some patients, this is the only way to regain their mental health and well-being.

\section{Spiritual intelligence and pain symptom management}

In 1998, the results of study conducted by McNeill et al, found that an inverse relationship between current pain intensity and general level of pain with overall satisfaction with pain management. Oral pain medications, prayer, intravenous and intramuscular injections were the top-ranked methods. Yates et al [31] mentioned that religion seems to be an important source of support for many patients. In addition to patients with advanced cancer who found comfort from their religious and spiritual beliefs were more satisfied, happier, and had less pain. Kaldjian, Jekel, and Friedland [9] found that spiritual beliefs and religious practices appeared to play a role in end-of-life decisions among patients with HIV. Thus Health-care providers need to recognize patients' spiritual beliefs and incorporate them into discussions about terminal care. The study of Cohen et al [32] found that there was a significantly relationship between meaningful existence subscale and a single item scale rating overall quality of life. In the same direction the results of the study conducted by Brady et al [8] found a significant correlation between spirituality and quality of life.

All these findings illustrate that spiritual intelligence play a role in coping with illness, and in turn its reliefs the symptoms pain. Thus, if we need to recover the homeostasis of patients, it is necessary to include the treatment plan developing the spiritual skills and meaning of life of these patients.

This is confirmed by the findings of a study conducted by Harris et al [34] that provided empirical evidence that recipients with strong beliefs who participated in religious activities had better physical and emotional well-being, fewer health worries, and better medical compliance by the final 12-month assessment. 
These findings suggest the development of specific nursing, social-service, or pastoral-involvement strategies, continuing staff education about the role of religion in patient care.

Spiritual intelligence helps patients to recover their physical and psychological, especially patients with chronic pain, high blood pressure, headaches, and irritable bowel syndrome. Ehman et al [35] found that $45 \%$ of participants reported that religious beliefs would influence their medical decisions if they become gravely ill. Ninety-four percent of individuals with such beliefs agreed or strongly agreed that physicians should ask them whether they have such beliefs if they become gravely ill. Forty-five percent of the respondents who denied having such beliefs also agreed that physicians should ask about them. Altogether, two thirds of the respondents indicated that they would welcome the study question in a medical history, whereas $16 \%$ reported that they would not. Only $15 \%$ of the study group recalled having been asked whether spiritual or religious beliefs would influence their medical decisions.

Keramati and Gutkin [36] said that survival requires living organisms to maintain their physiological integrity within the environment, which means they must preserve homeostasis. In this sense, efficient behavioral decisions depend on two brain circuits working in concert: the hypothalamic homeostatic regulation (HR) system, and the cortico-basal ganglia reinforcement learning (RL) mechanism.

Shapiro [19] argued that "our biological systems are designed with both positive and negative feedback loops that respond to the impact of the world around us. Negative feedback loops promote homeostasis, keeping things more or less as they are. We notice something is sharp (negative) and we pull back from it so we are not cut. Positive feedback loops promote novelty and growth. We notice that exercise makes us feel healthy (positive) and we begin to take regular walks. Both feedback loops are in continuous operation. Both are necessary. And she mentioned homeostasis is critical to survival. If our bodies do not maintain themselves within certain tolerances (temperature being one example) we cannot function. Growing through creative change is also critical. If we cannot adapt to shifts in our environment, we cannot function either. Ignoring the presence of change may give us a temporary sense of stability, but it is an illusion.

Cannon [37] referred that the adrenal medulla releases the hormone epinephrine into the blood when individual response to a perceived threat. Adrenal has a coordinating role involving many different body systems. In his theory for stress, Selye [38] pointed out that when homeostasis was challenged to the point of failure, the "thermostat of defense" needs to be raised to a higher level, by defensive mechanisms that stimulate the physiologic adaptive mechanisms.

Selye has been shown that continuous exposure to stress leads to disturbances in different parts of the body, leading to the emergence of the purposes called the general symptoms of dysplasia and this is happening in three stages: warning, resistance, exhaustion.

\section{The first phase: alarm}

At this stage, the body will call all its defensive forces to face the danger it is exposed to as a result of the sudden exposure to stimuli that was not prepared for it. It is a group of organic chemical changes. The blood sugar rises, the pulse accelerates, and the arterial pressure rises, thus the body is alert in order to adapt to the threatening actor.

\section{The second phase: resistance stage}

This phase includes the physical symptoms caused by the constant exposure to the stimuli and stress positions that the organism has acquired the ability to adapt to. This stage is important in the origin of the purposes of adaptation or the socalled cycosomatic purposes This occurs especially when the human ability to cope with situations cannot be met by adaptive reaction, and continuous exposure to pressure leads to an internal disturbance, resulting in more hormonal secretions causing organ disturbances.

\section{The third stage: exhaustion}

Exhaustion is likely to occur If the individual is exposed to multiple pressures for a longer period, he will reach a point unable to continue the resistance and enter the stage of exhaustion and becomes unable to fully adapt at this stage collapse hormonal payments and reduce the resistance of the body and damage many nerve devices and the patient goes to death at a rapid pace many adaptive responses that help the individual to protect themselves whenever exposed to changes and stressful situations are stopped. Low or increased temperature, hunger, thirst, excessive muscle activity and emotional stress all result in changes in the organism due to stress state.

Fletcher [39] mentioned that "Cannon originated the term homeostasis to describe the tendency of organisms to maintain stability or uniformity in their body states. By an extension of the principle, it might be used to describe the already demonstrated tendency to maintain status at the mental level of behavior, even in anticipation of the disturbing conditions. Instances of the usefulness of the term are drawn from such fields as perception (constancy), habit formation, learning, reasoning, work level (level of aspiration), and personality adjustment, to explain such mechanisms as rationalization and compensation" (p.80).

Through this view, biological homeostasis achieves the mental and psychological homeostasis of the individual, as well as the individual possessing the skills of spiritual intelligence achieves the physiological homeostasis and allostatic processes in the various functions of the body.

Thus Shapiro argued that "how do we develop our capacity to be open to the possibilities around us, to loosen our fear of impermanence? The first step is simply to acknowledge that we are working with the balance between homeostasis and creative change - that we need both. The second step is to pay attention not label, not judge, not push away, not grab - just pay attention to what is happening within us and around us right now. Seems 
simple enough, but actually it's not. Paying attention takes practice. Possibilities arise around us in every moment. We miss them because we are not paying attention, because we have labeled things before, we take the time to discover what they are. Someone looks different or speaks in a way we don't understand and, without noticing, we instantly give them a label ("old", "young", "beautiful", "unkind", "dangerous") and think we know something about them and what they bring to our lives. But we don't. Until we actually pay attention to what or who is in front of us, we really have no idea what gifts this experience may bring".

From the above view of Shapiro, we can say that Spiritual intelligence led to homeostasis, because all of this steps that develop our capacity to be open to the possibilities around us; working with the balance between homeostasis and pay attention to what is happening within us and around us right now, are include spiritual intelligence skills. This is consistent with Shapiro [40] said that as we train our attention, we can turn that basic curiosity to everything, rather than making judgments, we can simply become aware of what life is bringing us on all levels. And we can cultivate an appreciative awareness of the balance in our lives between homeostasis and creative change. Cannon considered homeostasis as part of the "wisdom of the body." Which is released by the integrity of body, mind, soul and spirit all together. As mentioned in Lambert these illustrations from Bernard and Cannon, attest to the body's wisdom for maintaining homeostasis-that is, a constant internal environment. The maintenance of homeostasis depends on a negative feedback system, a self-correcting process that reduces the discrepancy between a desired state and an actual state. Lambert mentioned a desired state is a condition crucial for life, comfort, or safety. The actual state of the system is compared to the set point, and if a discrepancy is detected, a self-correcting process is initiated. The process ends when the discrepancy reaches zero.

\section{Future Directions in the use of Spiritual Intelligence}

At present, with the proliferation of physical competition in the world, the speed of changes has increased, issues such as intelligence appeared more serious, especially the subject of spiritual intelligence as the infrastructure of individual beliefs, its capital of an important role in many areas, especially in mental and psychological health.

The development of interest in spiritual methods in psychotherapy is an appropriate attitude, and spiritual subjects have significant and significant effects on changes in mental health, Spiritual and religious values may facilitate the process of therapeutic progress. Spiritual intelligence is a key element in the achievement of the individual aims. The individual's possession of high spiritual intelligence is indicative of the individual's possession of other intelligences, and a clear indication of the individual's enjoyment of mental health and psychological homeostasis.

Todres, et al. [41] revealed that unique clinical pastoral education program provides the clinician with knowledge, language, and understanding to explore and support spiritual and religious issues confronting critically ill patients and their families.
Thus, they emphasized that incorporating spiritual care of the patient and family into clinical practice is an important step in addressing the goal of caring for the whole person.

Vaughan [42] emphasized that spiritual intelligence is associated with mental health in general, although some forms of spirituality may be dysfunctional or sick. When spiritual beliefs promote denial and rejection and lead to fear and conflict, they can be destructive and seriously problematic. Seybold and Hill [38] suggested that spiritual beliefs, practices and commitments are associated with positive outcomes, such as mental and physical health; marital satisfaction and stability; positive interaction with individuals; and a better quality of life. While Emmons [7] argued that one of the factors contributing to such positive outcomes may be that having a spiritual orientation to life protects people from undesirable and non-adaptive behaviors such as destructive, social or even personal behavior.

As for the importance of emotional intelligence in the health of individuals, the studies showed that high emotional intelligence was associated with high physical and mental health, high satisfaction with life, and emotional intelligence plays an important role in regulating health care. Spiritual intelligence plays an important role in human physical and psychological health. Studies have shown that spirituality has been positively correlated with adaptive ability in individuals with physical health problems and has also been associated with effective positive coping methods in cancer patients. Kirby et al. [43] study found that spirituality also reduces the negative effects of older people. And Fry study revealed that the sense of value and life was associated with increased physical and mental health in the elderly. King [44] emphasized that spiritual intelligence plays an important role in all the steps of health care, especially its management, treatment and healing, as it leads to appropriate results which other abilities / intelligences cannot do. Health is a general word that reflects not only the body, mind and spirit, not pain and happiness, only the whole human existence and the universal view of man.

Thus, the results of previous studies confirm the importance of spiritual intelligence to the physical and psychological health of the individual so that his quality of life can be realized. Howard [45] pointed out that spiritual intelligence enables the individual to confront and solve life problems, and the individual has the qualities of compassion, humility, gratitude, and wisdom. Atkinson [46] argues that harnessing the power of spiritual intelligence is the key to purity, prosperity and happiness, and to creating paradise on earth, because the self alone is not enough to make a more merciful and stable future. Spiritual intelligence is the solution, which is simply the intelligent energy that creates life and the strong desire to exist and to make everything new [47-52].

\section{Conclusion}

From all mentioned above, by spiritual intelligence interventions and understand homeostatic mechanism we can achieve a homeostasis in the secretion of hormones, neurotransmitters, heart attacks, blood pressure can be changed by the autonomic 
nervous system and cell and organ functions becomes better after a defect has been caused by the problems and stress experienced by the individual in his lifespan. Cannon [37] defined homeostasis as physiological mechanisms that maintain relatively constant the variables related to the internal milieu of the organism. Thus the Biopsychosocial-Spiritual Model of Care is a vital for applied if we are need to help the patients in reducing their physical and psychological suffering and achieving a better life for them. We need to plan counseling and psychotherapy programs to development spiritual intelligence for all individual to be happier and well-being, and to release quality of life for all healthy and patients' individuals across different stages of growth.

\section{Acknowledgement}

None.

\section{Conflicts of Interest}

No Conflicts of Interest to be declared.

\section{References}

1. Arnout B (2019) Applications of positive psychology in counseling and psychotherapy. LAMBERT for Academic Publishing, Germany.

2. Arnout B (2016) Spiritual intelligence and psychological counseling between theory and practice. Cairo: The Anglo-Egyptian Library.

3. Amram Y, Dryer C (2008) The integrated spiritual intelligence scale (ISIS). development and preliminary validation scale Paper Presented at the116th Annual Conference of the American Psychological Association Boston 8: 1-45.

4. Arnout B (2007) Spiritual Intelligence and its Relationship to Personality Characteristics in Different Age Samples. Journal of the Faculty of Education. Banha University, Egypt 17(72): 125-190.

5. Buzan $\mathrm{T}$ (2007) The power of spiritual intelligence. 10 ways to tap into your spiritual genius. New York: taper Collins.

6. Arnout B (2008) Spiritual Intelligence and its Relationship to Quality of Life. Journal of the Modern Education Association 313-389.

7. Zohar D, Marshall I (2000) SQ: spiritual intelligence: The ultimate intelligence. New York, NY. USA: Bloomsbury.

8. Emmons R (2000) Is Spirituality an Intelligence? Motivation, Cognition and the Psychology of Ultimate Concern. International Journal for the Psychology of Religion 10(3): 3-27.

9. Ornstein R (1998) The right mind: Making sense of the hemispheres. Orlando FL, Harvest Power S, Lundsten L (Eds) Studies that compare type theory and left-brain/right-brain theory. Journal of Psychological Type 43: 22-28.

10. Gallese V (2003) The roots of empathy: The shared manifold hypothesis and the neural basis of inter subjectivity. Psychopathology 36: 171-180.

11. Hamer D (2004) The God Gene. New York: Anchor Books.

12. Lazar S, Kerr C, Wasserman R, Gary J, Greve D, et al. (2005) Meditation experience is associated with increased cortical thickness. Neuro report 16(17): 1893-1897.

13. Singer W, Gray CM (1995) Visual feature integration and the temporal correlation hypothesis. Annual review of Neuroscience 18: 555-586.

14. Deacon T (1997) The symbolic species. London: Allen Laine.

15. Fry L (2003) Toward a theory of spiritual leadership. The Leadership Quarterly 14(6): 693-727.

16. Lutz A, Greischar L, Rawlings N, Ricard M, Davidson R (2004) Long-term meditators self-induce high-amplitude gamma synchrony during mental practice. Proc Natl Acad Sci U S A 101(46): 16369-16373.
17. Sulmasy D (2002) A biopsychosocial-spiritual model for the care of patients at the end of life. The Gerontologist 42(3): 24-33.

18. Jain M, Purohit P (2006) Spiritual intelligence: A contemporary concern with regard to living status of the senior citizens. J Indian Acad Appl Psychol 32(3): 227-233.

19. Wigglesworth C (2011) Spiritual intelligence and why it matters.

20. Baldwin J (2005) Jean Piaget. In Key thinkers in linguistics and the philosophy of language.

21. World Health Organization (1995) WHOQOL-100. Facet definitions and questions. WHO Division of mental health and prevention of substance abuse, Geneva, Switzerland.

22. Foglio J, Brody H (1988) Religion, faith, and family medicine. J Fam Pract 27(5): 473-474.

23. Lazarus R, Folkman S (1984) Stress, Appraisal, and Coping. New York: Springer.

24. Oman D, Reed D (1998) Religion and mortality among the communitydwelling elderly. Am J Public Health 88(10): 1469-1475.

25. Williams D, Sternthal M (2007) Spirituality, religion and health: evidence and research directions. Med J Aust 186(10): S47-S50.

26. D Souza R (2007) The importance of spirituality in medicine and its application to clinical practice. Med J Aust 186(10): S57-S59.

27. Wilding C (2007) Spirituality as sustenance for mental health and meaningful doing: a case illustration. Med J Aust 186(10): S67-S69.

28. Winslow G, Wehtje-Winslow B (2007) Ethical boundaries of spiritual care. Med J Aust 186(10): S63-S66.

29. Gioiella M, Berkma B, Robinson M (1998) Spirituality and quality of life in gynecologic oncology patients. Cancer Pract 6(6):333-338.

30. Koslander T, Da Silva A, Roxberg A (2009) Existential and spiritual needs in mental health care: an ethical and holistic perspective. J Holist Nurs 27(1): 34-42.

31. Yates J, Chalmer B, James P, Follansbee M, Mc Kegney F (1981) Religion in patients with advanced cancer. Med Pediatr Oncol 9(2): 121-128.

32. Cohen S, Mount B, Strobel M, Bui F (1995) The McGill Quality of Life Questionnaire: a measure of quality of life appropriate for people with advanced disease. A preliminary study of validity and acceptability. Palliat Med 9(3): 207-219.

33. Brady M, Peterman A, Fitchett G, Mo M, Cella D (1999) A case for including spirituality in quality of life measurement in oncology. Psychooncology 8(5): 417-28.

34. Casar Harris R, Amanda Dew M, Lee A, Amaya M, Buches L, et al. (1995) The role of religion in heart-transplant recipients' long-term health and well-being. J Relig Health 34(1): 17-32.

35. Ehman J, Ott B, Short T, Ciampa R, Hansen-Flaschen J (1999) Do patients want physicians to inquire about their spiritual or religious beliefs if they become gravely ill. Arch Intern Med 159(15): 1803-1806.

36. Keramati M, Gutkin B (2014) Collecting reward to defend homeostasis: A homeostatic reinforcement learning theory.

37. Cannon W (1932) The wisdom of the body. New York: Norton.

38. Selye H (1956) The stress of life. New York: McGraw Hill.

39. Fletcher J (1942) Homeostasis as an explanatory principle in psychology. Psychological Review 49(1): 80-87.

40. Shapiro A (2011) Getting Out of the Way: The Balance Between Homeostasis and Growth. Psychological Today.

41. Todres I, Catlin E, Thiel M (2005) The intensivist in a spiritual care training program adapted for clinicians. Crit Care Med 33(12): 27332736.

42. Sulmasy D (2002) A biopsychosocial-spiritual model for the care of patients at the end of life. The Gerontologist 42(3): 24-33. 
43. Kirby S, Coleman P, Daley D (2004) Spirituality and well-being in frail and non-frail older adults. Journals of Gerontology, Series B: Psychological Sciences \& Social Sciences, 59: 123-129.

44. King B (2007) Rethinking claims of spiritual intelligence: A definition, model, \& measure. Unpublished master's thesis, Trent University, Peterborough, Ontario, Canada.

45. Howard B (2009) Spiritual intelligence and transformational leadership: a new theoretical framework, Journal of Curriculum and Instruction 3(2): 54-67.

46. Atkinson M (2012) Discover true happiness. The power of spiritual intelligence.

47. Davidson R, Kabat-Zinn J, Schumacher J, Rosenkranz M, Muller D, et al. (2003) Alterations in brain and immune function produced by mindfulness meditation. Psychosomatic Medicine 65(4):564-570.

48. Gardner H (1999) Intelligence reframed: Multiple intelligences for the 21st century. New York: Basic Books.

49. Kaldjian LC, Jekel JF, Friedland G (1998) End-of-life decisions in HIVpositive patients: the role of spiritual beliefs. AIDS 12(1): 103-107.

50. Mc Neill J, Sherwood G, Starck P, Thompson C (1998) Assessing clinical outcomes: patient satisfaction with pain management. J Pain Symptom Manage 16(1): 29-40.

51. Seybold KS, Hill PC (2001) The role of religion and spirituality in mental and physical health. Current Directions in Psychological Science 10: 21-24.

52. Vaughan F (2002). What is spiritual intelligence? Journal of Humanistic Psychology 42: 16-33. 